\title{
COHERENT POWER SERIES RING AND WEAK GORENSTEIN GLOBAL DIMENSION
}

\author{
NAJIB MAHDOU \\ Department of Mathematics, Faculty of Science and Technology of Fez, Box 2202, University S.M. Ben \\ Abdellah Fez, Morocco \\ e-mail:mahdou@hotmail.com \\ MOHAMMED TAMEKKANTE \\ Department of Mathematics, Faculty of Science, Box 1014, University Mohammed V-Agdal Rabat, Morocco \\ e-mail: tamekkante@yahoo.fr \\ and SIAMAK YASSEMI \\ Department of Mathematics, University of Tehran, and School of Mathematics, Institute for Research in \\ Fundamental Sciences (IPM), P. O. Box 19395-5746, Tehran, Iran \\ e-mail:yassemi@ipm.ir
}

(Received 15 February 2012; accepted 25 July 2012; first published online 25 February 2013)

\begin{abstract}
In this paper we compute the weak Gorenstein global dimension of a coherent power series ring. It is shown that the weak Gorenstein global dimension of $R[[x]]$ is equal to the weak Gorenstein global dimension of $R$ plus one, provided $R[[x]]$ is coherent.
\end{abstract}

2000 Mathematics Subject Classification. 13D05, 13D02.

1. Introduction. All rings are commutative with identity throughout this paper. Let $R$ be a ring, and let $M$ be an $R$-module. As usual, we use $\operatorname{pd}_{R}(M), \operatorname{id}_{R}(M)$ and $\mathrm{fd}_{R}(M)$ to denote, respectively, the classical projective dimension, injective dimension and flat dimension of $M$.

The global dimension (or global homological dimension) of ring $R$, denoted $\operatorname{gldim} R$, is a non-negative integer or infinity which is a homological invariant of the ring. It is defined to be the supremum of the set of projective dimensions of all $R$-modules. Similarly, the supremum of the set of flat dimensions of all $R$-modules is called weak global dimension and denoted $\operatorname{wdim} R$. Jøndrup and Small in [11] gave a connection between a weak dimension of a coherent power series ring over a commutative ring $R$ and the weak dimension of $R$. Let $R$ be a ring, and let $x$ be an indeterminate over $R$. If $R[[x]]$ is a coherent ring, then Jøndrup and Small showed that $w \operatorname{dim}(R[[x]])=\operatorname{wdim}(R)+1$.

In this paper, we give an extension of this result to the Gorenstein weak dimension. More precisely, we prove that the weak Gorenstein global dimension of $R[[x]]$ is equal to the weak Gorenstein global dimension of $R$ plus one, provided $R[[x]]$ is coherent.

In the following we recall the definitions of the Gorenstein global dimension and the weak Gorenstein global dimension.

The Gorenstein projective dimension is a refinement of the classical notion of projective dimension of a module in the sense that it is always less than or equal to 
the projective dimension and the equality holds when projective dimension is finite. It was introduced by Enochs and Jenda in [7] as a generalization of the G-dimension defined by Auslander-Bridger [1] some 20 years earlier. In [6] the Gorenstein injective dimension was introduced as a dual notion of Gorenstein projective dimension. To complete the analogy with the classical homological dimension, Enochs et al. [8] introduced the Gorenstein flat dimension.

Recall that an $R$-module $M$ is called Gorenstein projective if there exists an exact sequence of projective $R$-modules:

$$
\mathbf{P}: \cdots \rightarrow P_{1} \rightarrow P_{0} \rightarrow P^{0} \rightarrow P^{1} \rightarrow \cdots
$$

such that $M \cong \operatorname{Im}\left(P_{0} \rightarrow P^{0}\right)$ and the functor $\operatorname{Hom}_{R}(-, Q)$ leaves $\mathbf{P}$ exact whenever $Q$ is a projective $R$-module. The complex $\mathbf{P}$ is called a complete projective resolution.

The Gorenstein injective $R$-modules are defined dually.

An $R$-module $M$ is called Gorenstein flat if there exists an exact sequence of flat $R$-modules:

$$
\mathbf{F}: \cdots \rightarrow F_{1} \rightarrow F_{0} \rightarrow F^{0} \rightarrow F^{1} \rightarrow \cdots
$$

such that $M \cong \operatorname{Im}\left(F_{0} \rightarrow F^{0}\right)$ and the functor $I \otimes_{R}-$ leaves $\mathbf{F}$ exact whenever $I$ is an injective $R$-module. The complex $\mathbf{F}$ is called a complete flat resolution.

The Gorenstein projective, injective and flat dimensions are defined in terms of resolutions and denoted by $\operatorname{Gpd}(-), \operatorname{Gid}(-)$ and $\operatorname{Gfd}(-)$ respectively.

In [4], the authors proved the following equality:

$\sup \left\{\operatorname{Gpd}_{R}(M) \mid \mathrm{M}\right.$ is an $R$-module $\}=\sup \left\{\operatorname{Gid}_{R}(M) \mid \mathrm{M}\right.$ is an $R$-module $\}$.

They called the common value of the above quantities the Gorenstein global dimension of $R$ and denoted it by $\operatorname{Ggldim}(R)$. Similarly, they set

$$
{ }^{w G g l d i m}(R)=\sup \left\{\operatorname{Gfd}_{R}(M) \mid \mathrm{M} \text { is an } R \text {-module }\right\},
$$

which they called Gorenstein weak dimension of $R$.

2. Main results. The main results of this paper are stated as follows.

THEOREM 2.1. Let $R$ be a ring and let $x$ be an indeterminate over $R$. If $R[[x]]$ is a coherent ring, then

$$
{ }_{w} \operatorname{Ggldim}(R[[x]])={ }_{w} \operatorname{Ggldim}(R)+1 .
$$

Proof. First we show that $\mathrm{wGgldim}(R[[x]]) \geq{ }_{\mathrm{wGgldim}}(R)+1$. The short exact sequence

$$
0 \longrightarrow R[[x]] \stackrel{x}{\longrightarrow} R[[x]] \longrightarrow R \longrightarrow 0
$$

implies that $R \cong R[[x]] / x R[[x]]$. Since $R$ is finitely presented as $R[[x]]$-module, we have that $R$ is coherent (see [9, Theorem 4.1.1(1)].

If $\operatorname{wGgldim}(R[[x]])=\infty$, then there is nothing to prove. Therefore, we assume that $\operatorname{wGgldim}(R[[x]])=n<\infty$. Using [9, Theorem 1.3.3] and [10, Proposition 2.27], we have $\operatorname{Gpd}_{R[[x]]}(R)=\operatorname{pd}_{R[[x]]}(R)=1$ and so by [5, Theorem 7] we have that 
${ }_{\mathrm{wGgldim}}(R[[x]])=n \geq 1$. Now let $M$ be a finitely presented $R$-module. By $[9$, Theorem 2.1.8], $M$ is a finitely presented $R[[x]]$-module. Thus, by [9, Theorem 1.3.5] and [5, Theorem 7], $\operatorname{Ext}_{R}^{n}(M, R)=\operatorname{Ext}_{R[x]]}^{n+1}(M, R[[x]])=0$. Therefore, by [5, Theorem 7], ${ }_{w} \operatorname{Ggldim}(R) \leq n-1$ and so ${ } \operatorname{Ggldim}(R) \leq \operatorname{wGgldim}(R[[x]])-1$.

To show that $\mathrm{wGgldim}(R[[x]]) \leq \mathrm{wGgldim}(R)+1$, we may assume $\mathrm{wGgldim}(R)=$ $n<\infty$. Let $M$ be a finitely presented $R[[x]]$-module and consider a short exact sequence of $R[[x]]$-modules

$$
\text { (*) } 0 \longrightarrow K \longrightarrow P \longrightarrow M \longrightarrow 0 \text {, }
$$

where $P$ is a finitely generated projective $R[[x]]$-module. By [9, Theorem 2.5.1], $K$ is also finitely presented since $R[[x]]$ is coherent. If $M$ is projective, then $\operatorname{Gpd}_{R[[x]]}(M)=$ $\operatorname{pd}_{R[x]]}(M)=0$. Otherwise, $K \neq 0$. Thus, by the Nakayama's lemma, we deduce that $K \neq x K$. Moreover, by [9, Theorem 2.1.8], $K / x K$ is a finitely presented $R$-module. On the other hand, from the short exact sequence $(*)$ we have $\operatorname{Tor}_{1}^{R[[x]]}(K, R)=$ $\operatorname{Tor}_{2}^{R[x]]}(M, R)=0$ since $\operatorname{fd}_{R[[x]]}(R) \leq 1 . K$ is a submodule of a projective $R[[x]]-$ module $P$, and $P$ is a submodule of a free $R[[x]]$-module. The element $\mathrm{x}$ is regular on any free $R[[x]]$-module, therefore regular on $\mathrm{K}$. Thus, from [3, Theorem 5.1], $\operatorname{Gpd}_{R[[x]]}(K)=\operatorname{Gpd}_{R}(K / x K) \leq n$. Accordingly, by [3, Lemma 2.4], $\operatorname{Gpd}_{R[[x]]}(M) \leq$ $\operatorname{Gpd}_{R[[x]]}(K)+1 \leq n+1$. Consequently, by [5, Theorem 7], wGgldim $(R[[x]]) \leq n+1$. Thus, we have the desired equality.

Recall that a ring $R$ is called an arithmetical ring if every finitely generated ideal is locally principal. If $\operatorname{wdim}(R) \leq 1$, then $R$ is an arithmetical ring (see, for instance, [2]). So we lead to ask the following question: If $\operatorname{wGgldim}(R) \leq 1$, then is $R$ arithmetical ring? In the following result we give a partial answer to this question. Recall that a ring is called quasi-Frobenius if it is Noetherian and self-injective.

Proposition 2.2. Let $R$ be a quasi-Frobenius ring which is not a field. Then the following statements hold:

(1) ${ }_{\operatorname{wGgldim}}(R[[x]])=\operatorname{Ggldim}(R[[x]])=1$.

(2) If $R$ is local with maximal ideal $\mathfrak{m}$, then $R[[x]]$ is not an arithmetical ring.

Proof. (1) Using [4, Proposition 2.8 and Theorem 2.9], we have that $\operatorname{wggldim}(R)=$ $\operatorname{Ggldim}(R)=0$. Thus, by Theorem 2.1, $\operatorname{wGgldim}(R[[x]])=1$. On the other hand, $R$ is Noetherian and so $R[[x]]$ is also Noetherian. Therefore, by [4, Theorem 2.9], $\operatorname{Ggldim}(R[[x]])=1$.

(2) Assume that $R[[x]]$ is an arithmetical ring. Let $a$ be a non-zero, non-invertible element of $R$ and let $I:=a R[[x]]+x R[[x]]$. Since $R[[x]]$ is a local arithmetical ring, we have $I=P R[[x]]$ for some $P:=\sum_{i} a_{i} x^{i} \in R[[x]]$ (where $a_{i} \in R$ ).

We have $P \in I=a R[[x]]+x R[[x]]$, so $P=a Q_{1}+x Q_{2}$ for some $Q_{1}\left(:=\sum_{i} c_{i} x^{i}\right)$, $Q_{2} \in R[[x]]$. Hence, $a_{0}=a c_{0}$. On the other hand, we have $a=P Q$ for some $Q=$ $\sum_{i} b_{i} x^{i} \in R[[x]]$. Hence, $a=a_{0} b_{0}$. We claim that $b_{0} \in \mathfrak{m}$. If this is not the case, then $b_{0}$ is invertible in $R$ and so $Q$ is invertible in $R[[x]]$; hence, we may assume that $P=a($ since $a R[[x]]=P Q R[[x]]=P R[[x]]=I)$. But, $x \in I=a R[[x]]$ implies that $x=a \sum_{i} d_{i} x^{i}$ for some $d_{i} \in R$ and so $1=a d_{1}$. This implies that $a$ is invertible in $R$, which is a contradiction. Therefore, $b_{0} \in m$. Since $a_{0}=a c_{0}$ and $a=a_{0} b_{0}$, we have that $a=a b_{0} c_{0}$ and so $a\left(1-b_{0} c_{0}\right)=0$. But $1-b_{0} c_{0}$ is invertible in $R$ since $b_{0} c_{0} \in M$. Thus, $a=0$, a contradiction. Therefore, $R[[x]]$ is not an arithmetical ring as desired. 
ACKNOWLEDGEMENTS. Siamak Yassemi was supported in part by a grant from IPM No. 91130214. The authors would like to express their sincere thanks to the referee for their helpful suggestions and comments, which have greatly improved this paper.

\section{REFERENCES}

1. M. Auslander and M. Bridger, Stable module theory, Memoirs of the American Mathematical Society, No. 94 (American Mathematical Society, Providence, RI, 1969).

2. S. Bazzoni and S. Glaz, Gaussian properties of total rings of quotients, J. Algebra 310(1) (2007), 180-193.

3. D. Bennis and N. Mahdou, First, second, and third change of ring theorems for Gorenstein homological dimensions, Comm. Algebra 38(10) (2010), 3837-3850

4. D. Bennis and N. Mahdou, Global Gorenstein dimensions, Proc. Amer. Math. Soc. 138(2) (2010), 461-465.

5. J. Chen and N. Ding, Coherent rings with finite self-FP-injective dimension, Comm. Algebra 24(9) (1996), 2963-2980.

6. E. Enochs and O. Jenda, On Gorenstein injective modules, Comm. Algebra 21(1993), 3489-3501.

7. E. Enochs and O. Jenda, Gorenstein injective and projective modules, Math. Z. 220 (1995), 611-633.

8. E. Enochs, O. Jenda and B. Torrecillas, Gorenstein flat modules, Nanjing Daxue Xuebao Shuxue Bannian Kan 10 (1993), 1-9.

9. S. Glaz, Commutative coherent rings, Lecture Notes in Mathematics, vol. 1371 (SpringerVerlag, Berlin, Germany, 1989). 193.

10. H. Holm, Gorenstein homological dimensions, J. Pure Appl. Algebra 189 (2004), $167-$ $21-24$.

11. S. Jøndrup and L. W. Small, Power series over coherent rings, Math. Scand. 35 (1974), 